\title{
Caracterización del ABS Utilizado en Procesos de Manufactura Aditiva y el ABS Natural
}

\author{
Characterization of ABS Used in Additive Manufacturing Processes and Natural \\ ABS
}

\author{
L. Vargas-Henríquez iD
}

\begin{abstract}
Resumen-El estudio de las propiedades del termoplástico Acrilonitrilo Butadieno Estireno (ABS) requiere de la caracterización previa de las propiedades mecánicas, de manera que se pueda obtener un conocimiento inicial para modelar y predecir el comportamiento mecánico de piezas fabricadas por impresión 3D, o simplemente para comparar los cambios que se producen en el material durante su transformación en la fabricación de los modelos. Este artículo presenta un estudio comparativo del comportamiento mecánico de piezas fabricadas por la técnica de manufactura aditiva, modelado por deposición fundida (FDM), con respecto a dos tipos de materiales termoplásticos: el ABS Plus utilizado en la técnica de FDM y el ABS natural. Las variables que se estudian son la resistencia a la tensión, resistencia a la fluencia y la elongación. Los resultados muestran que las propiedades determinadas para las probetas fabricadas con ABS natural, presentan una variabilidad menor que las obtenidas con ABS plus. Las muestras ensayadas fabricadas con el ABS natural presentaron una rigidez mayor que el ABS plus, y se comprobó que tiene una mayor resistencia a la tensión. Además, se aplicaron técnicas de caracterización para obtener información sobre la morfología del material y verificar las características dadas por el fabricante.
\end{abstract}

Palabras Claves-Manufactura aditiva, Modelado por Deposición Fundida (FDM), Acrilonitrilo-Butadieno-Estireno (ABS), Comportamiento mecánico, Prototipado Rápido.

Abstract-The study of the properties of the Acrylonitrile Butadiene Styrene (ABS) thermoplastic requires the prior characterization of the mechanical properties, so that an initial knowledge can be obtained to model and predict the mechanical behavior of parts manufactured by 3D printing, or simply to compare the changes that occur in the material during its transformation in the manufacture of the models. This article presents a comparative study of the mechanical behavior of parts manufactured by the additive manufacturing technique, molten deposition modeling (FDM), with respect to two types of thermoplastic materials: ABS Plus used in the FDM technique and natural ABS. The variables studied are tensile strength, creep resistance and elongation. The results show that the properties determined for the specimens manufactured with natural ABS have a lower variability than those obtained with ABS plus. In general, the distance of the nozzle is the manufacturing parameter that most influences manufacturing in FDM. The samples tested made with natural ABS showed a stiffness greater than ABS plus, and it was found to have a higher tensile strength. In addition, characterization techniques were applied to obtain information on the morphology of the material and to observe the thermal behavior and verify the characteristics given by the manufacturer. The proposed methodology is a reference of interest in studies for the determination of the mechanical properties of polymeric materials manufactured using these technologies.

Index Terms - Additive manufacturing, Fused Deposition Modeling (FDM), Acrylonitrile-Butadiene-Styrene (ABS), Tensile behavior, Rapid prototyping.

\section{INTRODUCCIÓN}

T A tecnología de manufactura aditiva por deposición fundida, FDM, utiliza una amplia gama de materiales para la fabricación de los productos. El más utilizado es el terpolímero Acrilonitrilo-Butadieno-Estireno, ABS. El ABS es un material heterogéneo formado por una fase homogénea rígida y una elastomérica. El estireno y el acrilonitrilo forman un copolímero lineal (SAN) que se desempeña como matriz. El butadieno y el estireno también forman un copolímero lineal (caucho de BS) que actúa como el material de relleno [1]. La combinación de los dos copolímeros forma el ABS, que es una combinación excelente de esfuerzo, rigidez y dureza. La estructura representativa se muestra en la fig. 1.
Este manuscrito fue enviado el 28 de agosto de 2019 y aceptado el 09 de marzo de 2020. El presente artículo es un subproducto del proyecto de investigación "Optimización experimental del parámetro de proceso en la tecnología FDM sobre las propiedades mecánicas del ABS" avalado por el programa de Ingeniería mecánica de la Universidad del Atlántico.

L. Vargas-Henríquez, Docente de la Facultad de Ingeniería, Programa de Ingeniería Mecánica (Grupo de Investigación IMTEF) de la Universidad del Atlántico (Puerto Colombia, Colombia). (e-mail: lisandrovargas@mail.uniatlantico.edu.co). 

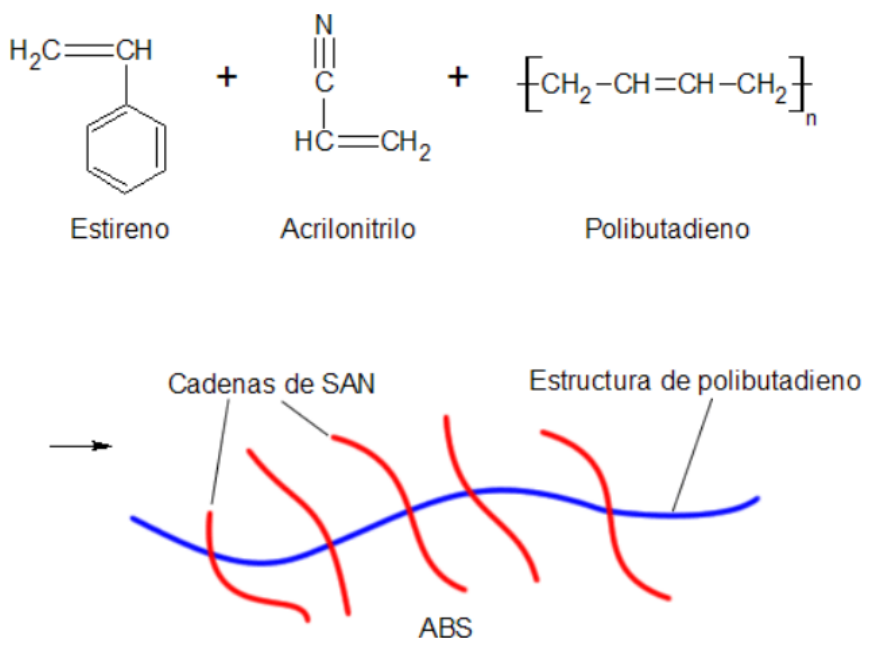

Fig. 1. Estructura química del Acrilonitrilo - Butadieno - Estireno [1].

Cada monómero es un componente importante del ABS: el acrilonitrilo, entre $15 \%$ y $35 \%$, contribuye con la resistencia a la temperatura, resistencia química y dureza superficial, mientras que el butadieno, entre $5 \%$ y $30 \%$, aporta dureza y resistencia al impacto, por último, el estireno, entre $40 \%$ y $60 \%$, contribuye con el brillo, rigidez y facilidad de procesamiento [1].

Dado el desarrollo de esta tecnología en los últimos años y la diversidad en las propiedades de los diferentes materiales utilizados en la tecnología FDM, la literatura disponible no es muy extensa. Sin embargo recientemente se han publicado una serie de trabajos dedicados a la caracterización mecánica de piezas producidas con FDM. Hossain [2] comprobó experimentalmente las características anisotrópicas en modelos de policarbonato fabricados por FDM, considerando parámetros de proceso como, el Airgap (espacio entre dos filamentos), la orientación y el ancho de la deposición y el ancho del contorno, concluyó que los modelos fabricados por FDM tienen resistencia a la tensión entre $65 \%$ y $72 \%$ que las piezas hechas por inyección y formularon una serie de recomendaciones de diseño para las piezas a ser fabricas con FDM. Tymrak [3] examinaron las propiedades a la tensión del $\mathrm{ABS}$ encontrando que la resistencia a la tensión de las piezas de ABS hechas en máquinas de FDM estaba en un promedio de 28.5 MPa. Wu [4] investigaron el proceso de extrusión del material teniendo en cuenta el gradiente de temperatura entre los filamentos contiguos, ya que estos permiten que los polímeros termoplásticos formen una interfaz de fusión sólida. Estableció que minimizar el airgap aumenta el área de contacto entre los filamentos depositados y conduce a una fusión más fuerte del material. Mohamed [5] analizaron los efectos de las condiciones del proceso sobre las propiedades mecánicas en función de la temperatura de piezas de PC-ABS fabricadas por FDM. Chacon [6] centra sus investigaciones sobre la influencia delos parámetros de fabricación sobre las propiedades mecánicas de las piezas de PLA. Todos los anteriores son de gran interés de la comunidad científica por aumentar el conocimiento sobre este tema.

A diferencia de otros trabajos, este artículo describe la caracterización de las propiedades mecánicas de modelos fabricados con el material termoplástico ABS Plus, en la maquina TItan SH-1 de FDM y se compara con las propiedades mecánicas del ABS natural. El conocimiento de las características del material utilizado en la manufactura aditiva FDM, permite a los fabricantes predecir las propiedades de las piezas finales y establecer pautas de diseño para su trasformación.

\section{MATERIALES Y MÉTODOS}

\section{A. Material para Manufactura Aditiva}

El ABS Plus (acrilonitrilo butadieno estireno) es un filamento de impresión 3D de uso común de $1,75 \mathrm{~mm}$ a $3 \mathrm{~mm}$ de diámetro y $15000 \mathrm{~cm}$ de largo. El termoplástico ABS Plus es de peso ligero, resistente al impacto y puede soportar condiciones climáticas extremas. Esto asegurará una envoltura o separación mínima de capas a medida que la pieza se enfría.

El ABS natural es conocido por su alta resistencia al impacto y resistencia última a la tensión. Tiene bastantes aplicaciones comerciales y de ingeniería, como por ejemplo, en tuberías, piezas automotrices, juguetes, electrodomésticos, etc. Por su alta resistencia a la temperatura es propenso a deformarse durante la impresión 3D. Esta es la razón por la cual una superficie de construcción calentada es crucial para la precisión dimensional. La comparación entre las principales propiedades del ABS Plus y el ABS natural se da en la Tabla I.

TABLA I

PROPIEDADES DEL ABS

\begin{tabular}{cccccc}
\hline \hline & $\begin{array}{c}\text { Resistencia a } \\
\text { la tensión } \\
(\mathrm{MPa})\end{array}$ & $\begin{array}{c}\text { Módulo de } \\
\text { elasticidad } \\
(\mathrm{GPa})\end{array}$ & $\begin{array}{c}\text { Resistencia } \\
\text { la impacto } \\
(\mathrm{J} / \mathrm{m})\end{array}$ & $\begin{array}{c}\text { Porcentaje } \\
\text { de } \\
\text { elongación } \\
(\%)\end{array}$ & $\begin{array}{c}\text { Densidad } \\
\left(\mathrm{kg} / \mathrm{cm}^{3}\right)\end{array}$ \\
\hline $\begin{array}{c}\text { ABS } \\
\text { Plus }\end{array}$ & 49 & 2,90 & 116 & 20 & 1,20 \\
\hline $\begin{array}{c}\text { ABS } \\
\text { Natural }\end{array}$ & $41-45$ & 2,34 & 210 & 25 & 1,04 \\
\hline \hline
\end{tabular}

La tabla I muestra los datos facilitados por el fabricante para los filamentos de los dos materiales utilizados. Como se puede observar, el ABS Plus es más rígido y tiene una mayor resistencia a la tracción, mientras que el ABS natural es un poco más dúctil. Sin embargo, la resistencia al impacto del ABS natural es mayor, $210 \mathrm{~J} / \mathrm{m}$ frente a $116 \mathrm{~J} / \mathrm{m}$.

\section{B. Diseño de las Probetas y Equipo de Manufactura}

Primero se creó un modelo CAD en el software Solidworks, de la probeta a utilizar para evaluar las propiedades mecánicas según la norma ASTM D638 Tipo IV para ensayos de tracción de materiales plásticos. La fig. 2, ilustra las dimensiones en milímetros utilizadas para crear el modelo CAD. 


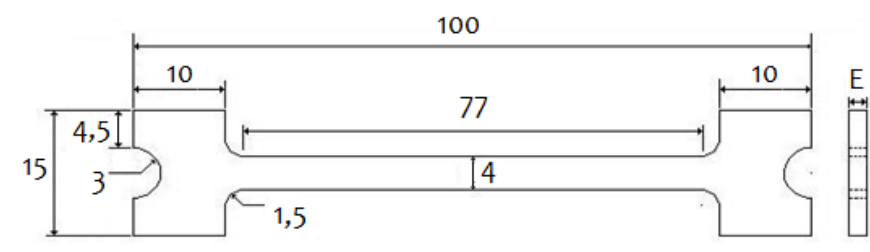

Fig. 2. Representación esquemática de la probeta plana para ensayo de tracción.

Luego, el archivo CAD se exporta al formato de estereolitografía (STL) y se importa al software de Insight de Stratasys el cual secciona o rebana el modelo en capas o planos horizontales con un grosor de capa de $0,025 \mathrm{~mm}$, como se muestra en la fig. 3. Se genera el código G que describe la trayectoria que debe recorrer la boquilla extrusora para depositar el material termoplástico hasta construir la geometría completa de la pieza. Las muestras se fabricaron utilizando el filamento de ABS Plus y ABS natural.

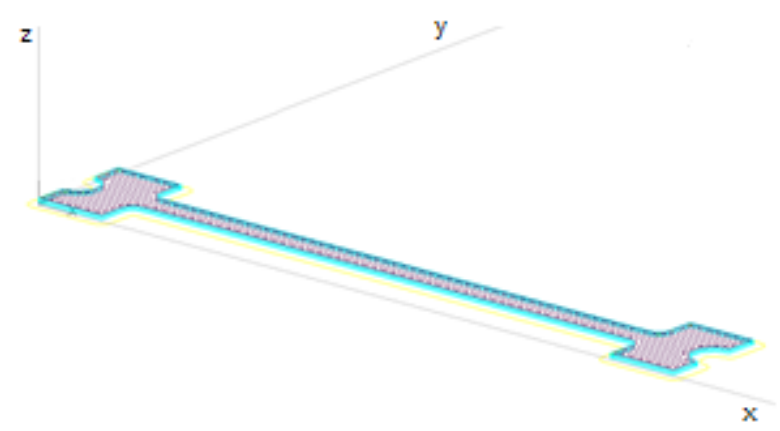

Fig. 3. Creación del recorrido y material de soporte para el Modelo.

Para la fabricación de las probetas en FDM se utilizó una impresora 3D modelo Titan SH-1 de la empresa Stratasys. Se seleccionó esta máquina por su flexibilidad en el ajuste de los parámetros de fabricación. Para tener resultados comparativos se dejaron fijos los parámetros de impresión mostrados en la tabla II.

TABLA II

PARÁMETROS DE PROCESO CONSTANTES PARA LA FABRICACIÓN DE LAS PROBETAS

\begin{tabular}{lc}
\hline \hline Parámetro & Valor \\
\hline Altura de la capa $(\mathrm{SI})$ & $0,254 \mathrm{~mm}$ \\
Ancho de la capa $\left(\mathrm{RW}_{\mathrm{p}}\right)$ & $0,508 \mathrm{~mm}$ \\
Altura y ancho del contorno & $0,508 \mathrm{~mm}$ \\
Recorrido de la boquilla & Zig-Zag \\
Forma del relleno & rectilínea \\
Temperatura de fusión & $240^{\circ} \mathrm{C}$ \\
Temperatura cama de impresión & $60^{\circ} \mathrm{C}$ \\
Diámetro de la boquilla & $0,40 \mathrm{~mm}$ \\
\hline \hline
\end{tabular}

Las características mecánicas de las piezas fabricadas por FDM dependen de los parámetros de fabricación. Las probetas se fabricaron con los siguientes parámetros de procesos, velocidades del cabezal de extrusión de $0,145 \mathrm{~mm} / \mathrm{s}$ y una distancia de la boquilla de deposición del material de 0,033 pulg $(0,84 \mathrm{~mm})$, este punto corresponde al setpoint de la máquina. La fig. 4 muestra un esquema del proceso FDM.

Velocidad del cabezal de extrusión: Es la velocidad con que se construye el prototipo, es decir la velocidad con la se deposita el filamento. Este factor afecta directamente la resistencia y la calidad de los prototipos, también incide sobre el tiempo de construcción y la exactitud dimensional.

Distancia de la boquilla de deposición: Este factor afecta al modelo debido a que se producen prototipos de densidades variables, También se produce ineficiencia en la adherencia entre capas y debido a la fluidez del material depositado se considera que hay mayor pandeo cuando la boquilla está más cerca de la plataforma de deposición. El material a mayor fluidez produce un redondeo fuera del efecto escalón que mejora el acabado final del prototipo [8].

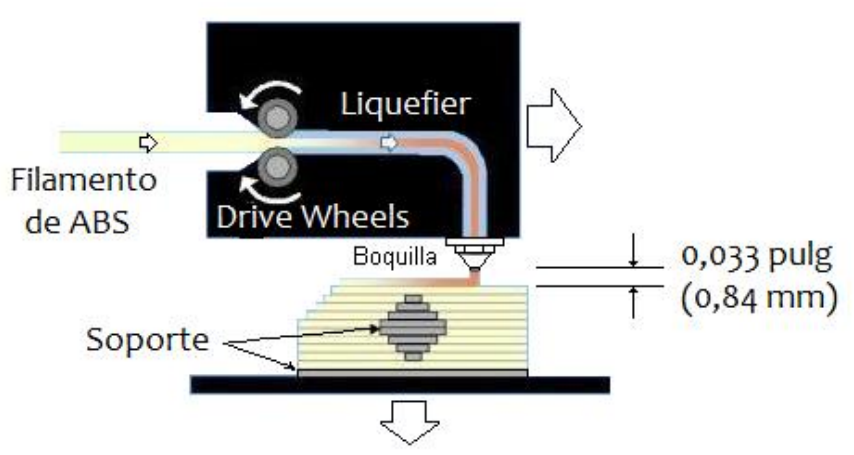

Fig. 4. Setpoint de Referencia para la Máquina Titán SH-1 de FDM [7].

\section{Pruebas Mecánicas}

Se realizaron pruebas de tensión a las probetas fabricadas con los dos materiales para comparar los datos con los obtenidos por el fabricante. Los ensayos mecánicos permiten determinar los valores promedios de los valores de la resistencia última a la tensión, el módulo de elasticidad y el porcentaje de elongación. Se compararon con los proporcionados por el fabricante.

La medición de las propiedades mecánicas se realizó en una máquina Stress/Strain de PASCO referencia AP-8216, siguiendo las recomendaciones de la norma ASTM D 638. El Sensor de Fuerza (Economy Force Sensor) CI-6746, alcanza una carga máxima es de $250 \mathrm{~N}$, y el Sensor de Movimiento Rotacional (Rotary Motion Sensor) CI-6538 contiene un encoder óptico que da un máximo de 1440 cuentas por revolución (360 grados) del eje del sensor. Por lo tanto, la velocidad de carga del ensayo fue de 4000 pasos/seg, generando un torque de $14 \mathrm{~N} / \mathrm{cm}$ y una velocidad lineal de $0,15 \mathrm{~mm} / \mathrm{s}$. El esfuerzo y la deformación son calculados usando el software DataStudio.

\section{Técnicas Empleadas para la Identificación del Material}

El método espectroscópico de difracción por rayos X, se empleó para obtener información sobre la morfología del ABS. Se utilizó un difractómetro Bruker D8 Advanced con geometría de difracción Bragg-Brentano, con un barrido entre $5^{\circ}$ y $100^{\circ}$. 
Para determinar la composición molecular de la muestra se utilizó el método de Espectrometría Infrarroja por Transferencia de Fourier (FT-IR) y el equipo disponible es el FT-IR Spectrum BX (Perkin Elmer). El equipo tiene grabado una base de datos y los espectros de los polímeros más importantes comercialmente, por lo tanto, se puede realizar identificación cualitativa de un polímero desconocido por comparación.

Para la caracterización superficial se realizó un análisis por Microscopia Electrónica, SEM utilizando un microscopio electrónico Philips ESEM XL 30 TMP y por Microscopia de Fuerza Atómica, AFM, se obtuvieron imágenes topográficas con el Microscopio de Barrido por Sonda, Park Scientific Instruments AutoProbe CP, en el modo Contacto (AFM - C).

Se realizó una prueba de Calorimetría Diferencial de Barrido (DSC) y de termogravimétrico (TGA) para observar el comportamiento térmico del ABS y verificar las características dadas por el fabricante.

\section{RESULTADOS Y DISCUSIÓN}

\section{A. Análisis Comparativo del Comportamiento Mecánico}

La fig. 5 muestra los resultados de las pruebas de tracción para las probetas de ABS Plus fábricas con velocidad del cabezal de extrusión de $0,145 \mathrm{~mm} / \mathrm{seg}$ y distancia de la boquilla de deposición de $0,84 \mathrm{~mm}(0,033$ pulg), distancia que corresponde al setpoint de la máquina.

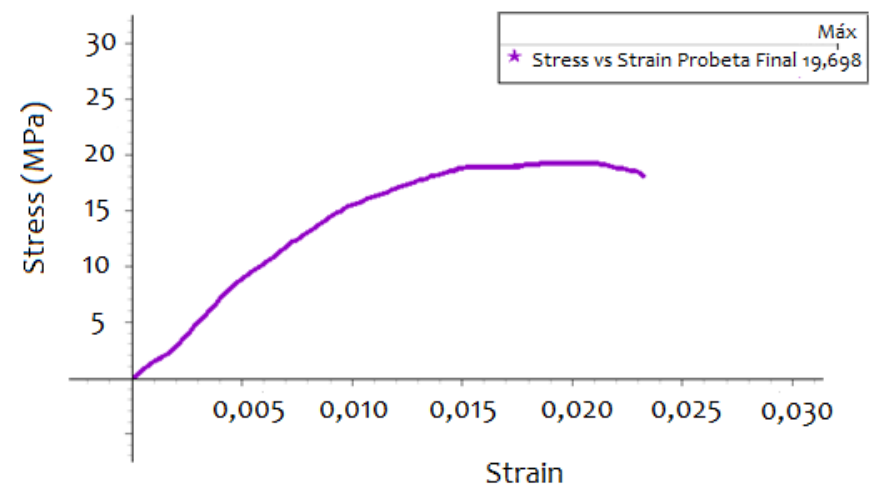

Fig. 5. Gráfica Esfuerzo-Deformación de las probetas de ABS plus fabricadas.

La fig. 6 muestra la curva de esfuerzo-deformación realizada para el filamento de ABS natural antes de ser extruido. Se observa que el material es rígido, resistente y muy tenaz, ya que presenta un módulo de elasticidad alto $(1893,47 \mathrm{MPa})$ y el área debajo de la curva es grande.

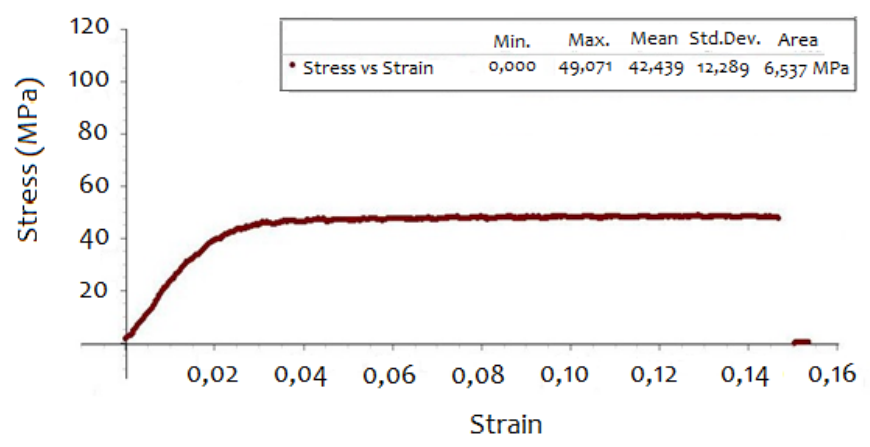

Fig. 6. Curva Esfuerzo-Deformación para el filamento de ABS natural.

La variabilidad de los resultados de las propiedades mecánicas puede ser comparada para ambos materiales a través de la tabla III.

TABLA III

COMPARACión de las Propiedades MeCÁNICAS del Material ABS PluS Y ABS NATURAL

\begin{tabular}{lcc}
\hline \hline Propiedades & ABS plus & ABS natural \\
\hline Resistencia a la Tensión (MPa) & 19,698 & 49,071 \\
Resistencia a la Ruptura (MPa) & 18,519 & 42,439 \\
Módulo de Elasticidad (Mpa) & 1450.360 & 1843,97 \\
Deformación Máxima (\%) & 0,022 & 0,15 \\
Desplazamiento (mm) & 1,969 & 7,010 \\
\hline \hline
\end{tabular}

Comparando los datos obtenidos experimentalmente con los aportados por el fabricante, se observa que existe una variabilidad significativa en el ABS plus con respecto al ABS natural.

Existe una variación en el comportamiento a la tracción de las muestras ensayadas del material ABS Plus, siendo su comportamiento general el más frágil en comparación con el filamento natural, que presenta un alto grado de ductilidad. La pendiente del área elástica disminuye claramente después de extruido el material, es decir cuando se modifica la orientación de fabricación.

Por otro lado, a la luz de los valores obtenidos, las piezas fabricadas con el filamento ABS natural tienen un mayor esfuerzo a la rotura que el ABS plus. Esto es debido al proceso de extrusión del material y por el calentamiento de este. Lo que genera una mayor ductilidad en el filamento de ABS natural.

Finalmente, las diferencias del ABS plus utilizado en el proceso FDM con respecto al material de partica, ABS natural, es de aproximadamente del 35\% menos. Esta disminución en las propiedades mecánicas se presenta debido a los poros que se forman en proceso de relleno de las capas (fig. 7), generando un menos volumen en la fabricación. 


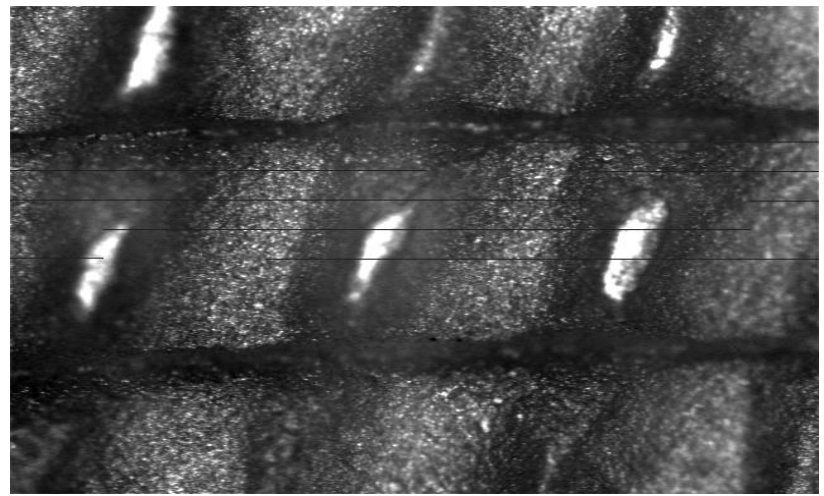

Fig. 7. Poros formados entre los caminos en los modelos fabricados por el proceso FDM.

\section{B. Difracción de Rayos $X$}

En la difracción de rayos $X$ realizada al filamento de ABS Plus, en un barrido entre $5^{\circ}$ y $30^{\circ}$ se observan picos significativos a $13,5^{\circ}, 16,1^{\circ}$ y $19,8^{\circ}$. Realizando una comparación de la difracción del filamento de ABS natural con el del ABS plus (fig. 8), se observa que el filamento de ABS presenta una morfología similar a la del ABS de referencia con excepción del pico a $7,06^{\circ}$, que es producto del alineamiento de las cadenas moleculares durante el proceso de fabricación del material.

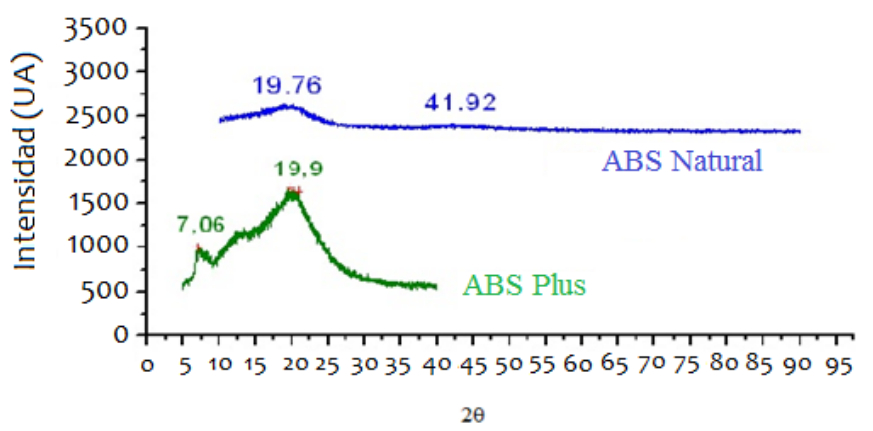

Fig. 8. Difracción comparando el filamento de ABS natural y el ABS Plus.

\section{C.Microscopia Electrónica de Barrido (ESEM) y} Espectroscopia de Energía Dispersa de Rayos X (EDS).

La fig. 9 muestra un espectro EDS obtenido sobre la muestra de ABS natural, así como una micrografía ESEM del mismo lugar donde se obtuvo el EDS.

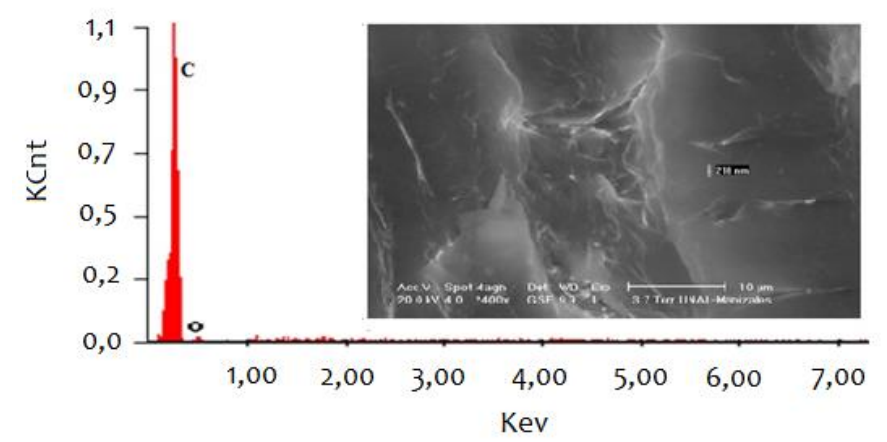

Fig. 9. Espectro EDS e imagen ESEM para el ABS natural.
Se observa que la muestra es completamente homogénea, con una fuerte composición de carbono.

La fig. 10 muestra el EDS obtenido sobre la el ABS Plus.

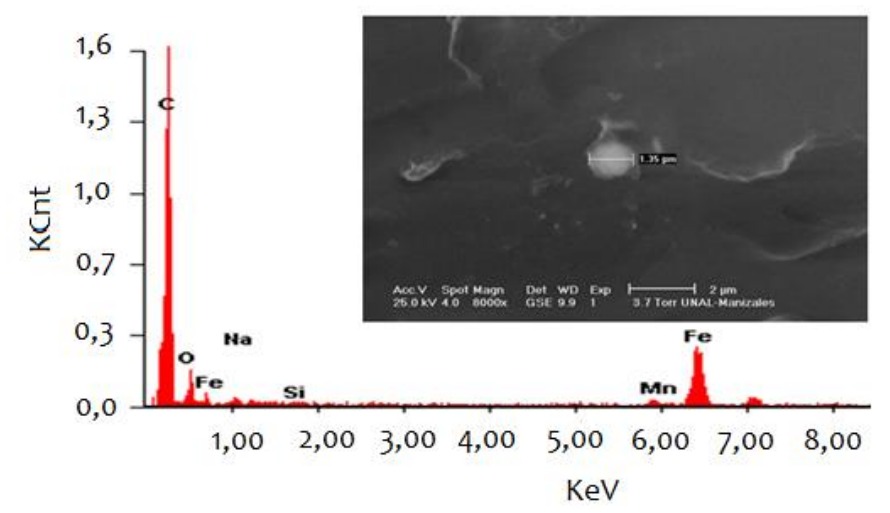

Fig. 10. Espectro EDS e imagen ESEM para el ABS plus.

En el ABS Plus se encontró un hueco o poro en la microestructura del material de aproximadamente $1,35 \mu \mathrm{m}$. Y además los picos de hierro, silicio y manganeso encontrado corresponden al sustrato utilizado, por lo tanto, al eliminar esos picos se observa que la estructura es homogénea con un pico significante de carbono como el del ABS natural.

\section{D.Microscopia de Barrido por Sonda (SPM)}

La fig. 11 muestra la imagen topográfica de la muestra de ABS Plus en diferentes áreas con barrido de $40 \mu \mathrm{m} 2$.

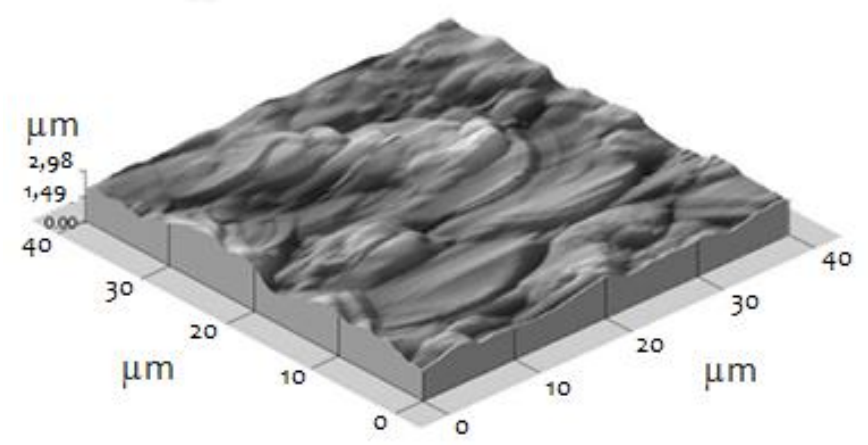

Fig. 11. Imagen topográfica del material ABS Plus.

En la imagen se puede observar un cierto grado de orientación de la estructura presentada por el proceso de fabricación del material, este tipo de orientación no se puede prevenir completamente.

\section{CONCLUSIÓN}

En este trabajo se ha estudiado la comparación de las propiedades mecánicas de los termoplásticos ABS Plus, utilizado en procesos FDM, y el ABS natural. Los resultados muestran una disminución en las propiedades mecánicas del ABS Plus de aproximadamente $35 \%$ al ser extruido en el 
proceso con respecto al ABS natural.

En general, las piezas fabricadas con ABS natural se comportan de forma más rígida que en el caso del ABS Plus. Sin embargo, no hay una tendencia clara en cuanto a la influencia sobre el módulo de elasticidad, a diferencia del ABS natural que mostró un mayor grado de ductilidad que el ABS plus.

La máxima resistencia a la tensión de 49,071 $\mathrm{MPa}$ se obtiene para las probetas fabricadas con el ABS natural. Y de igual manera presenta un mayor porcentaje de elongación de 0,15 .

Todas estas disminuciones de las propiedades mecánicas de debe a los huecos o poros encontrados en la microestructura del material producto del proceso de relleno del material capa por capa. También se debe a las tensiones internas que se generan en la interfaz entre capas, es decir, la zona más débil de las piezas fabricadas utilizando manufactura aditiva.

En general, se puede decir que el porcentaje de relleno es un factor de mayor influencia en los resultados.

La metodología propuesta es una referencia de interés para estudios de las propiedades mecánicas de los materiales poliméricos fabricados por FDM. Específicamente estos resultados pueden ser muy útiles para la selección de los materiales y parámetros adecuados en los procesos de diseño y fabricación de FDM.

Como una línea de trabajos futuros, se propone la inclusión de la variabilidad de los factores, velocidad de extrusión y la densidad, así como la influencia de las interacciones de estos factores en los resultados. Además, la influencia de otros parámetros avanzados que están estrechamente relacionados a fenómenos termofísicos y/o químicos más específicos (la temperatura local del filamento durante la deposición, la difusión molecular en la interfaz del polímero y la presión/fuerza de deposición, entre otros).

\section{REFERENCIAS}

[1] Billmeyer Jr W.F. “Ciencia de los Polímeros". España, Editorial Reverte, pp 259 - 300. 2004.

[2] Hossain M. S, Ramos J, Espalin D, Perez M, Wicker R. "Improving tensile mechanical properties of FDM-manufactured specimens via modifying build parameters". 24th. Annual International Solid Freeform Fabrication Symposium: an additive manufacturing conference, pp 380-392. Texas. 2013.

[3] Tymrak, B.M., Kreiger, M. \& Pearce, J.M. "Mechanical properties of components fabricated with open-source 3-D printers under realistic environmental conditions". Materials and Design, 58, pp.242-246. 2014. DOI:10.1016/j.matdes.2014.02.038.

[4] Wu W, Geng P, Li G, Zhao D. Zhang H, Zhao J. "Influence of Layer Thickness and Raster Angle on the Mechanical Properties of 3D-Printed PEEK and a Comparative Mechanical Study between PEEK and ABS". Materials, 8, 5834-5846. 2015. DOI: 10.3390/ma8095271.

[5] Mohamed O. A. Masood S. H. Bhowmik J. "Analytical Modelling and Optimization of the Temperature Dependent Dynamic Mechanical Properties of Fused Deposition Fabricated Parts Made of PC-ABS". Materials 9(11), 895. 2016. DOI: 10.3390/ma9110895.

[6] Chacón J.M. Caminero M.A. García-Plaza E. Núñez P.J. "Additive manufacturing of PLA structures using fused deposition modelling: Effect of process parameters on mechanical properties and their optimal selection". Material \& Deign. 124, 143-157. 2017. DOI: 10.1016/j.matdes.2017.03.065.

[7] L. Vargas Henríquez, E. Córdoba Nieto. "Inspección de la Calidad Superficial en el Prototipado Rápido". Editorial Académica Española, pp 20-25. 2015.
[8] Bharath Vasudevarao, Drama Prakash Nataraja. "Sensitivity of RP Surface Finish To Process Parameter Variation". Department of Industrial Engineering, Arizona State University. 2000.

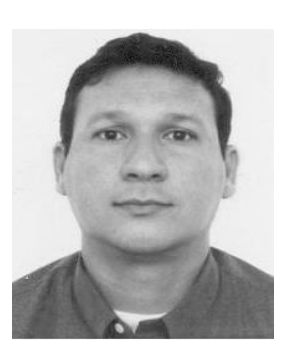

Lisandro Vargas Henríquez. Ingeniero Mecánico de la Universidad del Atlántico, Barranquilla, Colombia y Magister en Ingeniería - Materiales y Procesos de la Universidad Nacional de Colombia, Bogotá, Colombia; Actualmente es estudiante del Doctorado en Ciencia y Tecnología de los Materiales de la Universidad Nacional, Bogotá,

Colombia.

Desde el 2007 hasta 2014, fue coordinador del programa de Ingeniería Mecánica en la Universidad del Atlántico. Docente Investigador de la Universidad del Atlántico. Miembro del Grupo de Investigación IMTEF (Ingeniería de Materiales, Procesos y Tecnologías de Fabricación), con experiencia investigativa en las áreas de Prototipado Rápido, Procesos de Maquinado, Desarrollo de Productos y mantenimiento ORCID: https://orcid.org/0000-0002-0967-957X 